




\section{Sommaire}

\section{L'imaginaire des médias \\ Sous la direction de Michel Maffesoli et Moisés de Lemos Martins}

Introduction. À propos de l'imaginaire des médias

Michel MAFFESOLI, Moisés de Lemos MARTINS

\section{Images et imaginaire}

Dreamland. La ville comme ruine et comme mémoire Alberto ABRUZZESE, Antonio RAFELE

Médias et mélancolie - le tragique, le baroque et le grotesque Moisés de Lemos MARTINS

Limage récréative : des photos fantaisistes aux jeux virtuels Maria da Luz CORREIA

Inconscient collectif et noosphère

Raphaël JOSSET

Transe et réalité virtuelle. L'homo religiosus à l'ère des nouvelles technologies
Frédéric VINCENT

\section{Nouvelles images techniques} La flamme électronique. Quand la masse se fait avant-garde
Vincenzo SUSCA

Techno-œil : jeux de formes et d'images

Fabio LA ROCCA

Médias localisés, territoire informationnel et mobilité

André LEMOS

Éloge des liaisons techniques

Jean-Martin RABOT

Pour comprendre les nouvelles liaisons digitales :

le concept d'individuation chez Carl digitales :

José PINHEIRO NEVES 


\section{Pratiques de l'image}

Médiations technologiques dans l'espace urbain contemporain Julieta LEITE

L'esthétique de l'écoute. Sur la liaison de l'imaginaire radiophonique à la parole émotive Madalena OLIVEIRA

Poétiques du générique de cinéma : l'expressionnisme en mouvement Nelson ZAGALO

De comment est (re)fait l'espace-entre

A Cidade dos Objectos [La Ville des Objets] (Augusto Alves da Silva) Helena PIRES

Plis et fragments. La turbulence des sens dans la publicité automobile Albertino GONÇALVES

Les images numériques s'imaginent l'archaïque :

mettre en perspective les cartes postales

Moisés de Lemos MARTINS,

Madalena OLIVEIRA \& Maria da Luz CORREIA

\section{Activités sociologiques}

Underground Zone. Dandyn Punk, Beautiful People de Claudia Attimonelli, Antonella Giannone par Vincenzo SUSCA

La quête de reconnaissance, nouveau phénomène social total de Alain Caillé (éd.)

Par Vincent SEVEAU 


\title{
ÉlOGE DES LIAISONS TECHNIQUES
}

Jean-Martin RABOT *

\begin{abstract}
Résumé : Du point de vue d'une sociologie du quotidien et de l'imaginaire, les nouvelles
technologies ne peuvent pas être envisagées comme source d'aliénation sation. Elles ne conduisent pas à l'annihilation due source d'aliénation ou d'homogénéipotentialités imaginatives de l'homme (Ellul) $\mathrm{Ni}$ discours ontologique (Freund) ou des (Heidegger), dans le but de mobiliser l'h (Ellul). Ni même à "l'arraisonnement " de l'ètre les technologies sont avant tout vecteurs de socialité simple objet technique. Les nouveldéveloppement technologique est parfaitement logique (Durand) et le retour aux valeurs compatible avec le réinvestissement mythoques qui se développent au sein des réseaux informautaires (Maffesoli). Les liaisons technirelations et d'interactions sociales.
\end{abstract} Mots clés : technique, nouvelles technologies, médias interactifs, réseaux, imaginaire, so-
cialité.

\begin{abstract}
From the point of view of the sociology of everyday life and of the imaginary, new technologies cannot be seen as a source of alienation or homogenisation. They do not potentialities of man (Ellul) Nor even to speech (Freund) or either of the imaginative aiming at mobilizing men as simple technical "enframing of the being" (Heidegger), when tor of sociality. In addition, in post-modeal objects. New technologies are primarily a veccompatible with the mythological reinvestment, the technological development is perfectly ian values (Maffesoli). The technical systems networks are therefore producers ofions that are developed within the information
\end{abstract}

Keywords : technique, new technologies, interactive media, networks, maginary, sociality.

\footnotetext{
Centre d'Études de Con Portugal.jmrabot@ics.uminho.pt
} 


\section{Technique et modernité}

Dans un premier temps, nous exposerons la conception de la technique pendant la modernité, en prenant comme exemple la philosophie de Jacques Ellul, et dans un second temps nous porterons notre attention sur la signification que les nouvelles technologies prennent dans la postmodernité.

Même pendant la modernité, une période caractérisée par la croyance dans la suprématie de la raison, la technique a été liée à l'imaginaire. Pour pouvoir se développer, la technique s'est nourrie de l'imaginaire faustien, qui stipule la possibilité d'un progrès continu et simultané dans toutes les sphères d'activité sous l'égide de l'action concertée de la grande majorité des hommes, toujours dotés de raison et de bonne volonté. C'est au moyen du mythe et comme croyance que le progrès a été conçu en tant que progrès technique: "Cette puissance spirituelle qu'exerce l'idée de progrès, c'est son efficacité symbolique, composante des croyances magiques. Même "fatalisé", le progrès, en tant qu'objet de croyance, engage ceux qui y croient à collaborer au mouvement par lequel il est censé advenir. Il suffit pour cela de vouloir ce qui est censé ne pas pouvoir ne pas advenir. De vouloir le "bon" mouvement, le mouvement nécessaire, à savoir le perfectionnement de l'humanité dans tous les ordres. La technique assure son effectivité à cette volonté, elle la redéfinit comme l'expression d'un désir de maitrise qui ne cesse de toujours mieux se satisfaire ${ }^{1}$. "

C'est donc aux domaines du mythe et de la croyance que l'on doit les espérances placées dans la possibilité d'une résolution définitive des conflits. On se souviendra que Marx a fait du progrès technique la condition première de l'accès de l'homme à la conscience de son exploitation, à la conscience de soi et à la conscience de l'inexorabilité du changement. On se souviendra surtout des critiques acerbes que Schmitt proférera à l'encontre de ceux qui voyaient dans la technique la panacée aux conflits inhérents à l'ère industrielle que les socialistes saint-simoniens, les marxistes ou encore les libéraux associaient pourtant au pacifisme. La réflexion de Schmitt sur les neutralisations peut nous aider à prendre conscience des méfaits que ces illusions portent à la compréhension et même à la résolution des conflits. Selon Schmitt, les hommes, au cours de leur histoire, ont échafaudé l'idée selon laquelle il leur serait socialement utile et bénéfique de neutraliser une activité déterminée pour en faire une source de pacification de l'ensemble de la société. Ce rôle de neutralisation a successivement été dévolu à la théologie qui, au Moyen Âge et surtout au XVIe siècle, a décrété la trêve de Dieu, en reléguant les croyances dans le domaine du privé, pour suppléer aux guerres de religion, puis au XVII siècle à la métaphysique, avec le développement des sciences de la nature, ensuite à la morale humanitaire au XVIIIe siècle, qui voyait dans la vertu et dans l'éducation en tant que méthode de diffusion de la vertu le moyen de concilier l'homme et la raison, et enfin au XIXe et au XXe siècle à l'économique puis à la

1. P.-A. Taguieff, Le sens du progrès. Une approche historique et philosophique. Paris, Flammarion, 2004, p. 93.

Sociétés $n^{\circ} 111-2011 / 1$ 
technique, au point que la confiance placée en eux finissait par relever de la religiosité magique : "la religion qui croit au miracle et à l'au-delà se mue directement

En consonance avec ce mouvement, le mythe, en particulier quand il s'agit du mythe politique (nous pensons aux analyses de Georges Sorel et de Jean-Pierre Sironneau), peut contenir une prétention à la vérité. Mais, comme l'a constaté justes " que dans la prétentions à la vérité, chimériques et inauthentiques, ne sont technique " 3 .

Comme il est bon de voir, cette conception de la technique ne se suffit pas à elle-même. La technique ne peut avoir raison de tout. Si la finalité - et surtout la prétention de la technique - est " d'éliminer toutes les formes d'inconnu ", comme l'a montré Umberto Galimberti, il nous faudra également reconnaitre avec lui que l'opération mathématique ssoudre l'inconnu dans l'inconnue d'une équation que prométhéenne qui signifie "que même de résoudre " 4 . Il s'agit là d'une croyance instant nous pourrions, pourvu seus savons ou que nous croyons qu'à chaque n'existe en principe aucune puissancent que nous le voulions, nous prouver qu'il dans le cours de la vie; bref que nous pouvonseuse et imprévisible qui interfère sion " 5 . La volonté manifeste " "de prendrens maîtriser toute chose par la prévides fins "spirituelles" " 6 n'est au plus qu'une man" la technique et l'orienter vers crit encore dans cette croyance prométhéenne.

Nous en viendrons maintenant

Ellul sur la technique Celle-ci est à exposer brièvement la réflexion de Jacques çue. Ellul part du présupposé est typique de la manière dont la modernité l'a conavec le pluralisme politique, que le totalitarisme n'était pas seulement compatible nourrissait : "Il va de soi que lorsque ne, culturel ou médiatique, mais qu'il s'en couches sociales. Il est bien évident que identité dans tous les pays et toutes les fie ce sont des modes d'agir et même d'être, maique se spécifie, que ce qu'elle uniun autre genre de vie que les pays froids, et quais que les pays chauds obligent à l'unité fondamentale de la société technicienne les nationalismes subsistent malgré pas les grandes avenues rectilignes d'identité . Le monde technicien n'entraîne sité apparente peut régner pourvu qu'entité des idéologies! La plus grande diver-

2. C. Schmitt, La notion de politique. Théorie du partisan. Paris, Calmann-Lévy, 1972,

3. C. Kerényi, "Mythe et technique ", in Revue Diogène. Une anthologie de la vie intel4. U. Galimberti, Psiche e techne 2005, pp. 113-130, p. 118 pour la citation. Paulus, 2006, pp. 424 et 425 .

6. M. Heidegger, "La question de la technique Union Générale d'Éditions, 1974, p. 70. mard, 1988, pp. 9-48, p. 11 pour la citation.

Sociétés $n^{\circ} 111-2011 / 1$ 
sous le pluralisme apparent des formes culturelles se précise un système universel donc fallacieux en ce tous lieux ${ }^{7}$. " Le pluralisme politique idéologique serait cien.

La technique serait le fait fondamental auquel les sociétés postindustrielles auraient à se plier. Ainsi, l'homme " reste parfaitement capable de choix, décision, modification, orientations... Mais toujours à l'intérieur du cadre technicien et dans dent en ce sens : "le système la technique " 8 . Toute une série de remarques abonchamp de possibles, mais exclusivement semble donner à l'homme un plus large encore : "le système technicien est de l'humain dans la Technique plus en plus humanisé. Mais par l'absorption d'idées à une politique technicié . On aura donc affaire dans le même ordre cisé, etc. Rien ne peut s'ón ce que parce que les tentatives à l'emprise de la technique sur l'homme, ne seraitpeuvent que revêtir une foutives d'échappatoire ou d'opposition à la technique ne Technique ne peut être que composécienne. Ainsi, " un État apte à dominer la que obéissant aux impératifs de lasé de techniciens "11. Pareil sort est fait à l'éthiqu' " une éthique vécue dus la technique. Aussi ne peut-elle être autre chose fonctionne bien " 12 . À l'instar exigé pour que le système technicien également l'imaginaire : "Le système les autres activités, la technique se soumet lui-même en système symbolique. À l'echnicien est un univers réel qui se constitue un univers imaginaire un reflétégard de la nature, l'univers symbolique était rapport à ce monde naturelet sur-ordonné, entièrement institué par l'homme par férencier de cette réalité symbolique qui attribuait un même temps maitriser le réel par la médiation du tème technicien, il n'y a plus aucune pou monde par ailleurs indifférencié. Dans le sysparce que le réel est produit par l'hossibilité de symboliser, en ce sens-là, d'abord tère et d'étrangeté. Il se prétend toujours, qui n'éprouve pas le sentiment de myssi la symbolisation est un processusours directement le maître. Ensuite, parce que est au contraire un mécanisme d'in de distanciation, tout le processus technique tenant ce n'est plus l'homme qui symbégrion de l'homme. Enfin, parce que mainsymbolise elle-même (...). La symbolisation une nature, mais la technique qui se cien 13 . "

C'est dans cette optique qu'Ellul a abordé les relations entre la technique et la religion, dans un livre publié antérieurement. Selon l'éminent philosophe, les mou-

7. J. Ellul, Le système technicien. Paris, Calmann-Lévy, 1977, pp. 209-210.
8. Ibid., p. 360.
9. Ibid., p. 122.
10. Ibid., p. 129.
11. Ibid., p. 147.
12. Ibid., p. 163.
13. Ibid., p. 195.

Sociétés n ${ }^{\circ} 111-2011 / 1$ 
" vements culturels et artistiques, à l'exemple du jazz aux États-Unis, ou encore les

d'" un débordement ins " qui se manifestent par un appel à la libération au moyen une folie de l'action, sociale et politique 14 , peuvent parfaitement être et mer, dés dans leur composante religieuse de reliance et parfaitement être appréhentions. Néanmoins, ces mouvements neliance et esthétique de partage des émotrahir, leur profonde inscription temps, Ellul affirme le caractère insf la civilisation technique. Dans un premier autant de formes d'opium du peuple. Mais de ces mouvements, qui représentent renforcer la domination de peuple. Mais dans un second temps, ils ne font que que dans une société non technicienne, Le jugement est alors sans appel : « alors nels et particuliers d'adoration, dans ce nouveau dejets très divers, parfois personorientation. Les objets seconds sont éliminés, toutes milieu il n'y a plus qu'une seule utilisées, il n'y a plus de "fuites", et leur condensation produit des rent et sont extatiques comme il n'y en eut jamais pour l'ampleu produit des phénomènes donc pas un hasard si les phénomènes ex l'ampleur et la durée. (...) Ce n'est tés les plus techniques. Il faut s'attendrextatiques se sont développés dans les sociésens. Ceci n'a d'autre signification que la contraire, à un accroissement dans ce la technique. (...) L'extase est soumise au moumission de la vie religieuse nouvelle à

Ellul est finalement amené à méprise monde technique et le sert 15. "

techniciste, une société qui banalise l'artificiatíé rationalité à l'homme dans sa totalité, tandificialité en imposant ses exigences de satisfaisait d'une application régionale. C'est que précédemment toute activité se nique qu'Ellul condamne. La notion de milieu a le caractère totalitaire de la techSelon l'éminent philosophe, l'homme a vécu dans troinit Ellul à parler de système. son évolution : le milieu naturel dans la période préh milieux différents au long de la période historique, le milieu technicien dans préhistorique, le milieu social dans Ellul établit une distinction entre l'opération technique la post-historique. Ensuite, le système technique.

L'opération technique a accompagné toute l'évolution de l'homme ; le phénomène technique, quant à lui, existe depuis la fin de la période historique, et produit naturel pour le " phagocyter cancer dans le corps. Le passage de l'opéro prolifère dans la société à l'instar d'un que a eu pour conséquence la destruction des technique au phénomène techninelles. Ce processus a suscité l'association des propriétés des techniques traditiona produit la société technicienne. L'éconon des différentes techniques modernes et ces de la technique et il ne resta plus à l'homie et l'État se sont adaptés aux exigenveau qui lui était dévolu. Le raisonnement d'Ell que de se conformer au rôle nouparce qu'il nous montre que le processus d'exlul n'en reste pas là, ne serait-ce que milieu naturel et social par la technique est expropriation puis d'appropriation du

14. J. Ellut, La tect

15. Ibid., pp. 384 et 385.

Sociétés $n^{\circ} 111-2011 / 1$ 
que elle-même se constitue en simple moyen. Ce stade est précisément atteint dans les sociétés les plus avancées. En effet, la technique forme maintenant un écran entre l'homme, ses congénères et la nature. Elle est devenue " une forme générale d'intervention " 16 , à laquelle nous faisons obligatoirement appel pour rester en rapport avec tous ces éléments. En somme, de nos jours, tout passe par la médiation de la technique qui exclut toutes les autres médiations, y compris celle de la symbolisation.

L'homme finit alors par être complètement aliéné, séparé du monde réel qui l'entoure, comme de lui-même. Nous nous engageons ainsi sur la voie du divertissement continu, c'est-à-dire sur « la voie du détournement de l'homme et de son addiction par la société technicienne, par la fascination. Il nous faut prendre divertissement, non pas au sens d'amusement, mais au sens pascalien : l'homme est diverti, c'est-à-dire, d'une part, détourné de penser à soi-même, à sa condition humaine, et aussi détourné des plus hautes aspirations, du sens de la vie, des objectifs supérieurs $» 17$.

Si Ellul voit dans la théorie de l'information et des ordinateurs l'élément moteur qui permet de comprendre le passage de la société technicienne au système technicien, auquel il donne également le nom de "technique technicisée ", nous pouvons cependant voir en eux une redécouverte et une réactualisation de la dimension tactile, mythologique, onirique et imaginaire de l'humanité.

\section{Nouvelles technologies et postmodernité}

S'il est totalement légitime de penser que la technique touche "à la racine de l'âme » 18 et qu'elle dépossède l'homme de toute vie intérieure, s'il est encore légitime d'envisager les techniques comme une forme d'octroi d'un nouveau moyen qui s'impose à l'homme, lui enlève toutes ses prérogatives et se présente à lui comme son seul horizon de vie possible, en déterminant et contraignant ainsi de manière panoptique sa manière de penser et d'agir, il n'est pas moins légitime de conjecturer qu'elle s'opère dans le sens d'un renforcement de l'interaction entre les hommes. Il est tout à fait vrai que le principe de la séparation et de la distanciation a été l'emblème de l'Occident dans tous les domaines: dans la religion, avec la conception d'un Dieu tout-puissant et la séparation entre le corps et l'âme; dans la science avec la rupture avec le sens commun et la séparation entre le sujet et l'objet. D'une façon analogue, la technique a souvent été appréhendée dans la seule perspective de sa fonctionnalité, de son instrumentalité, de sa performativité, en conduisant de plus en plus, comme nous l'indique Simmel au sujet de l'économie monétaire, à une séparation entre le monde objectif de la culture et le monde subjectif des personnes. C'est en ce sens que la sociologie critique a appréhendé la

16. J. Ellul, Le système technicien, op. cit., p. 44.

17. J. Ellul, Le bluff technologique. Paris, Hachette, 1988, p. 421.

18. G. Bernanos, Les grands cimetières sous la lune. Paris, Plon, 1988, p. 88.

Sociétés $n^{\circ} 111-2011 / 1$ 
technique : une corruption du concept aristotélicien de telos, de l'idée kantienne du royaume des fins, du principe horkheimerien d'une raison objective.

L'originalité des penseurs de la postmodernité réside dans le fait d'avoir mis un terme aux principes de séparation et de critique. Force est de constater que le développement de la technique renforce le sentiment d'appartenance tribale. C'est grâce aux réseaux communicatifs qui se constituent les réseaux sociaux. La " galaxie électronique ", dont parlait Moles, offre un espace d'expression aux différentes logiques sociétales, aussi bien aux logiques fonctionnelles qui se trouvent au fondement de tous les réseaux de communication qu'aux logiques érotiques ou ludiques. Comme l'a remarqué Patrice Flichy, dans son livre sur L'imaginaire d'Internet, l'écran constitue un lieu de prédilection " où les utopies peuvent prendre corps et être expérimentées "19. Même si, à la façon de Gilbert Simondon, nous pensons la technique en termes d'individuation, en termes de métamorphose de l'humain en du non-humain par le truchement de la technique, nous ne pouvons faire l'impasse sur ce qui relie la personne au champ pré-individuel ou trans-individuel
dans lequel celle-ci s'inscrit.

Louis Dumont avait défini l'idéologie moderne comme correspondant au primat du rapport aux objets sur le rapport aux hommes. La sociologie imaginale et objectale élaborée par Michel Maffesoli nous enseigne que les images et les objets reliance. Si les progrès techniques doivent être envisagés dans leur fonction de ment dans le dechnologiques suscitent des espoirs infondés, notamsouvent de prétexte de la génétique et de l'informatique, et qu'elles servent trop mentales, des représentations, millénariste [qui] fait toujours naître des images vécues comme des fictions " 20 , des fictions transformées en réalités, des réalités vêtrement de fiction et de réalité "le pas moins intéressant de voir dans cet enchede la technique le moteur de l'a "le paradoxal réenchantement du monde faisant la religion matérialiste du Moyen Âge avait annihilé 21 . De la même manière que l'âme, séparation Age avait annihilé la séparation entre le corps et siasme suscité par les ordinateurs, les téléphones portables, Inel déglise, l'enthouà l'opposition entre les sujets et les objets, opposition sur laquelle se fondait le poutermes d'Auguste Comte.

L'air du temps est à l'empathie généralisée avec les objets, une empathie qui nous renvoie immanquablement à l'intersubjectivité, à l'intercorporalité, à l'interactivité. En se rapportant à l'exemple de l'utilisation de la technologie comme magie, Richard Stivers a clairement mis en évidence le caractère de socialisation et de divinisation des ordinateurs. Même si la technologie venait à créer " dans son

19. P. Flichy, L'imaginaire d'Internet. Paris, La Découverte, 2001, p. 260 20. M. Onfray, Féeries anatomiques. Généalogie du corps faustien. Paris,
pp. 181-182.

La Table Ronde, 2004. p. 100 .

Sociétés $n^{\circ} 111-2011 / 1$ 
épanouissement une civilisation entière... l'attribution de magie à l'existence humaine " 22 représente une nécessité insurmontable de la condition humaine. Nous comprenons mieux ce qui unit les ordinateurs à la religiosité postmoderne : "Les techno-païens comprennent l'ordinateur comme étant une "machine magique". L'ordinateur est une forme de magie finale et plus puissante, elle récapitule toutes les formes précédentes. (...) L'ordinateur crée un univers d'informations et ce faisant il promeut une identification mystique à lui-même. Ses dévots deviennent des connaisseurs d'une information qui, à partir d'un certain niveau d'ingestion, provoque l'extase. Les techno-païens sont les grands-prêtres d'un culte selon lequel l'univers et ses divinités se trouvent dans l'ordinateur 23. "Cette remarque nous permet de comprendre que la réalité postmoderne est caractérisée par l'hénothéisme. Et c'est bien ce qui a attiré sur elle les foudres du pape actuel, Benoit XVI, qui au tout début de son pontificat avait procédé à l'élaboration d'une liste actualisée de péchés, plus adaptée aux temps nouveaux. Ainsi, sont considérés comme des péchés le fait de voir excessivement la télévision, le fait d'abuser des jeux électroniques, le fait de naviguer de façon exagérée sur la toile. Et s'ils le sont, c'est parce qu'ils représentent autant de formes qui concurrencent directement le monothéisme religieux.

Dans son ouvrage consacré à la technique, Andrew Feenberg s'est exprimé sur les possibilités de détour des nouvelles technologies et d'échappatoire aux finalités instrumentales pour lesquelles ces technologies ont été conçues. À cet égard, il a fait appel à la notion de "flexibilité interprétative de la technique " pour nous signifier qu' " un enchaînement de dispositifs dont la configuration avait été pensée comme la solution à un problème déterminé - la distribution d'informations - fut appréhendé par ses utilisateurs comme la solution à un problème tout à fait différent : la communication humaine "24. Les moyens de communication actuels constituent précisément le creuset à partir duquel les groupes se forment, se consolident et se séparent mutuellement. Des groupes qui à chaque fois ont des contours différents, des objectifs divers, des styles de vie variés. Des groupes qui peuvent être durables ou éphémères, ouverts ou fermés, élargis ou restreints. Des groupes qui en tout cas nous rappellent la horde de Fourier, la structure clanique ou totémique de Durkheım, la tribu de Maffesoli. Des groupes soudés et reliés par des affinités électives et sélectives.

De ce fait, l'utilisation massive et même incontrôlée des téléphones portables ou de l'Internet n'a pas mis un terme au goût pour l'errance, et elle a même ravivé la communication au niveau planétaire. Cette communication généralisée est trop souvent le prétexte d'une critique qui, invoquant la crise de l'humain en raison du déplacement dans « la civilisation occidentale de la parole vers l'image, d'un territoire

22. R. Stivers, A tecnologia como magia. O triunfo do irracional. Lisboa, Instituto Piaget, 2001, p. 14.

23. Ibid., p. 15

24. A. Feenberg, (Re)penser la technique. Vers une technologie démocratique. Paris, La Découverte, 2004, pp. 105-106.

Sociétés n $111-2011 / 1$ 

multiplicité par la dia/bolé " 25 , s'en tient au constat de l'appauvrissement de l'expépostmodernité ne saurait se complaire darice. En d'autres termes, une sociologie de la tion généralisée en termes de processus de perte de l'identité cette communicareconstruction au moyen d'un réseau mondial, c'est-à-dire et de sa nécessaire technique " qui relie de façon artificielle ce qui est donné de d'une " "matrice" ce qui dispersé dans l'espace et le temps en une donné de façon fragmentaire et oubliant qu'il n'existe de liaison sans déliaison une unité qu'elle veut indivisible en qui est en cours c'est l'appropriation de l'imaison : "dans le fond tout indique que ce liaison absolue et parfaite. Dans une l'imaginaire théologique et mythique d'une les choses, les images, les objets, les corps et primitivisme ultra-tech, tout est lié : dans la tension qui lie et délie, avec toutes et les machines. Or l'humain s'exprime

Du point de vue de la postmodernité, les nouvelles technósitations et saléatoire 26. " mystique et peuvent être comprises comme une les technologies renvoient à la Cette stylisation ne peut toutefois être prise une stylisation esthétique du social. traditionnelle, une construction artificielle comme corruption de la communauté socialisation "se faisant passer pour une Gemeinschaft d'une nouvelle forme de en fait bien plus analogue aux communautés esthétint à la Tönnies, mais qui est et maintenues en vie principalement, peut-être uniquement, Kant, mises au monde dévotion de leurs membres » 27. Faut-il peut-être uniquement, par l'intensité de la l'enthousiasme (mouvement intrinsèque) rappeler que cette dévotion résulte plus de tions (mouvement extrinsèque). Et nous le constatons au jour le jour : le recours à photoblogs, aux vidéoblogs s'inscrit dans au courrier électronique, aux blogs, aux pice à la création de temps et de territoires ambiance communicationnelle prosocialité. L'utilisation de générations sures symboliques dans lesquels s'incarne la d'iPhone (smartphones), d'iPad (tablettes numériqued (baladeurs numériques), cité de fonctions ou plutôt de fonctionnalités, comme l'on disposant d'une multiplil'appareil photo, la caméra, le baladeur, le client Internet qui dit aujourd'hui, comme le Web ou de consulter les courriers électroniques, lnternet qui permet de naviguer sur vocale visuelle, le téléchargement d'applications diverses allant texte, la messagerie sociaux, en passant par les GPS, la télévision, les livres, les journaux, les magazines,
les films, les musiques, ou encore les bandes dessinées, sionifie, d'une part, meture sur soi et une coupure avec le monde et, d'auées, signifie, d'une part, une ferAcciaiuolo, M.; Babo, M. A. (Ed) Arte melancolia: o trágico, o barroco e o grotesco". Estudos de Arte Contemporânea \& Centro de Estudos Lisboa, Instituto de História da Arte/ 2011, pp. 53-65, p. 56 pour la citation. 26. J. Bragança de Miranda, Parion.

Miranda \& M. T. Cruz, Crítica das Ligaçōes na Era da Técnoçes técnicas ", in J. Bragança de

pp. 259-277, p. 269 pour la citation.
27. Z. Bauman, La vie en miettes. Expérien na da Técnica. Lisbonne, Tropismos, 2002 ,

Chambon, 2003, p. 171.

Sociétés $n^{\circ} 111-2011 / 1$ 
partielle de la relation sociale sur la base d'une affirmation ou d'une revendication de l'appartenance à une marque, en l'occurrence Apple. Nous aimerions rappeler que la marque MacIntosh constitue le point de jonction et de reconnaissance mutuelle de nombreux intellectuels français. Le préfixe "i", le "je" des différents Pod, Phone et Pad n'est plus à subsumer sous la catégorie de l'identité de l'individu ; il renvoie bien au " "Je est un autre" rimbaldien [qui] n'est plus, simplement, une exagération poétique, mais une "hyperréalité" se démultipliant en des myriades d'exemplaires $» 28$.

L'importance actuelle que revêt Internet est paradigmatique de la postmodernité, dans la mesure où elle est le signe d'une intensification des communions humaines. Ainsi, nous pouvons lire dans la prolifération de forums de discussion ou de rencontres, de jeux, l'indice d'un fusionnement des êtres fondé sur le partage des émotions. Les médias interactifs en sont le meilleur des exemples. On peut lire dans la propagation des images (iconiques, publicitaires, télévisées, virtuelles) une remagification du monde (Tacussel), une "reliance généralisée " (Morin) qui contribuent à la renaissance de l'imaginaire sociétal. Cette reliance est d'autant plus marquante et profonde qu'elle se manifeste sur le terrain de la technique qui se veut pourtant neutre, source de rationalisation, dans un monde objectivant et objectivé et néanmoins constitué de connexions, de links, de on-line.

Tous ces liens techniques, caractérisés par " l'accélération anachronique de la réalité présente " 29 qui consacre l'immédiateté, l'instantanéité, la simultanéité et fait perdre à l'homme jusqu'à la conscience de sa nécessaire inscription dans le temps et l'espace, conduisent cependant à un approfondissement des relations de proximité. Dans la conception de Paul Virilio, "l'addiction, la dépendance compulsive à Internet et à ses si nombreux moteurs de recherche, est une première réponse à l'origine de cet effet de réel où l'interactivité amène déjà certains fidèles à quitter leur environnement concret, à vider les lieux d'une vitalité organique et sociale »30. En fait, dans cette optique, la prolifération des images correspond à une désertion des lieux, à un oubli du temps historique, qu'il appartienne au passé, au présent ou au futur. L'internaute ne vit donc que par concaténation d'instants aussi futiles que passagers échappant à l'histoire et à l'attribution d'un quelconque sens historique. Plutôt que de se centrer sur les méfaits de l'accélération du temps, de la mobilisation permanente de l'iridividu, de la fabrication commerciale des émotions, il nous semblerait bien plus judicieux de comprendre " combien le festif, l'imaginaire, l'onirique collectif vont devenir les normes de l'espace "cyber" „31.

28. M. Maffesoli, "Un narcissisme tribal ", préface à H. Azuma, Génération Otaku. Les enfants de la postmodernité. Paris, Hachette Littératures, 2008, pp. 5-8, p. 7 pour la citation.

29. P. Virilio, Le futurisme de l'instant. Stop-Eject. Paris, Galilée, 2009, p. 69.

30. Ibid., p. 81 .

31. M. Maffesoli, "Cyberculture: "communion des saints" postmoderne ", in M.Z. Coelho (ed.), Não poupes no semear. Trinta anos de comunicação, Aníbal Alves. Coimbra, Pé de Página Editores, 2009, pp. 189-196, p. 191 pour la citation.

Sociétés $n^{\circ} 111-2011 / 1$ 
La métaphore baroque est à même de rendre compte de la navigation ou de la circumnavigation sur ce que l'on appelle le réseau ou la toile. Il ne s'agit pas tant de circuler linéairement d'un point de départ à un point d'arrivée, mais de passer de sites en sites selon le " modèle leibnizien du pli et du repli, du dépliement indéfini " 32 . Les sites sont comme des boîtes que l'on ouvre et que l'on referme, à l'exemple des matriochkas, ces poupées russes qui s'emboîtent les unes dans les d'emblée offert pourrait-il en être autrement quand on sait que ce qui nous est rend bien, ofter sur l'écran est l'ensemble du réseau, que la notion d'hypertexte tions raccordées qu'elle renvoie à l'existence d'un stockage sans limites d'informadéplient et se délient. elles par des liens. Des liens qui relient à mesure qu'ils se baroque des sculpture déploiement sans fin de liens sur nos écrans évoque le courbures en multiplient mouvement qui brouillent les distances, rapprochent les contraires et s'étendent à l'infines " 33 . On retrouve encore la métaphore baroque des plis qui réelle »34. Hier comi dans ce que Manuel Castells appelle « la culture de la virtualité sa forme bru comme aujourd'hui, l'expérience se présente à nous, non pas dans médiatisée par un confrontation directe et immédiate à la réalité, mais elle est communication unsemble de codes et de symboles. La spécificité du mode de celle dont les actuel réside dans le fait qu'il ne produit plus la réalité virtuelle, " construit la virtualité réelle en permanente attente d'actualisation, mais qu'il lequel la réalité même (c'est-à-direst-ce à dire? "Il s'agit d'un système dans entièrement captée, (c'est-à-dire l'existence matérielle/symbolique des gens) est de simulacres, dan immergée, dans un cadre d'images virtuelles, dans un univers où l'expérience est lequel les apparences ne se situent pas seulement sur l'écran donc l'ensember communiquée, mais deviennent l'expérience même 36. "C'est bolisée par les méd expérience humaine qui est happée puis représentée et syml'expérience, que la costeractifs. Faut-il alors penser que l'image se substitue à informatique) " 37 se submunication électronique (typographique, audiovisuelle ou ble le croire. Mais il faut bie aux autres formes de communication? Castells semse démultiplier et ce faisan reconnaître que cette expérience est aussi appelée à dans ce dépliement. C'est peute ne cesse de se déplier et de retrouver une unité chement des interfaces qu'il faut co dans ce dépliement de l'expérience par le trule retour aux valeurs communt comprendre le réinvestissement mythologique et

32. G. Chazal, "Internet : interface baroque ", in D. Parrochia (éd.), Penser les réseaux.

Seyssel, Champ Vallon, 2001, pp. 54-66, p. 63 pour la citation.

33. A. Gonçalves, Vertigens. Para uma sociologia da perversidade. Coimbra, Grácio Edi-

34. M. Castells, La société en réseaux. L'ère de l'information. Paris, Fayard, 2001, p. 468.
36. 469.

36. Ibid.

37. Ibid., p. 471.

Sociétés $n^{\circ} 111-2011 / 1$ 


\section{Bibliographie}

Bauman Z., La vie en miettes. Expérience postmoderne et moralité. Rodez, le Rouergue Chambom, 2003.

Bernanos G., Les grands cimetières sous la lune. Paris, Plon, 1988

Castells M., La société en réseaux. L'ère de l'information. Paris, Fayard, 2001

Chazal G., «Internet : interface baroque ». Parrochia D. (dir.), Penser les réseaux. Seyssel, Éditions Champ Vallon, 2001, pp. 54-66.

Ellul J., Le système technicien. Paris, Calmann-Lévy, 1977

Ellul J., La technique ou l'enjeu du siècle. Paris, Éditions Economica, 2001.

Ellul J., Le bluff technologique. Paris, Hachette, 1988.

Feenberg A., (Re)penser la technique. Vers une technologie démocratique. Paris, Éditions La Découverte, 2004.

Flichy, P., L'imaginaire d'Internet. Paris, Éditions La Découverte, 2001.

Galimberti U., Psiche e techne O homem na idade da técnica. São Paulo, Editorial Paulus, 2006.

Gonçalves A., Vertigens. Para uma sociologia da perversidade. Coimbra, Grácio Editor, 2009. Heidegger M., «La question de la technique », in Essais et conférences. Paris, Gallimard, 1988, pp. 9-48.

Kerényi C., "Mythe et technique ». Revue Diogène. Une anthologie de la vie intellectuelle au XXe siècle. Paris, PUF, 2005, pp. 113-130.

Maffesoli M. Le rythme de la vie. Variations sur les sensibilités postmodernes. Paris, Éditions de La Table Ronde, 2004.

Maffesoli, M. "Un narcissisme tribal ». Préface à H. Azuma, Génération Otaku. Les enfants de la postmodernité. Paris, Hachette Littératures, 2008, pp. 5-8

Maffesoli M., "Cyberculture: "communion des saints" postmoderne ". Coelho, M. Z (Org.), Não poupes no semear. Trinta anos de comunicação, Aníbal Alves. Coimbra, Pé de Página Editores, 2009, pp. 189-196.

Martins M. de Lemos, « Media e melancolia: o trágico, o barroco e o grotesco ». Acciaiuolo, M.; Babo, M. A. (Ed.) Arte e Melancolia. Lisboa, Instituto de História da Arte/Estudos de Arte Contemporânea \& Centro de Estudos de Comunicação e Linguagens, 2011, pp. 53-65.

Miranda, J. Bragança de, «Para uma critiqua das ligaçōes técnicas ». Miranda, J. Bragança de; Cruz, M. T., Crítica das Ligaçōes na Era da Técnica. Lisbonne, Tropismos, 2002, pp. 259-277.

Onfray M., Féeries anatomiques. Généalogie du corps faustien. Paris, Grasset, 2003.

Schmitt C., La notion de politique. Théorie du partisan. Paris, Calmann-Lévy, 1972.

Stivers R., A tecnologia como magia. O triunfo do irracional. Lisboa, Instituto Piaget, 2001.

Taguieff P.-A., Le sens du progrès. Une approche historique et philosophique. Paris, Flammarion, 2004

Virilio P., Le futurisme de l'instant. Stop-Eject. Paris, Galilée, 2009

Weber M. Le savant et le politique. Paris, Union Générale d'Éditions, 1974.

Sociétés $n^{\circ} 111-2011 / 1$ 